\title{
Spin induced gigahertz polarization oscillations in vertical-cavity surface-emitting laser devices
}

\author{
M. Y. Li ${ }^{* a}$, H. Jaehme ${ }^{a}$, H. Soldat ${ }^{a}$, N. C. Gerhardt ${ }^{a}$, M. R. Hofmann ${ }^{a}$ and T. Ackemann ${ }^{b}$ \\ ${ }^{a}$ Photonics and Terahertz Technology, Ruhr-University Bochum, Universitätsstrasse 150, ID04/327, \\ D-44780 Bochum, Germany; \\ bSUPA and Department of Physics, University of Strathclyde, Glasgow G4 0NG, Scotland, U. K.
}

M. Y. Li, H. Jaehme, H. Soldat, N.C. Gerhardt, M.R. Hofmann, and T. Ackemann. "Spin induced gigahertz polarization oscillations in vertical-cavity surface-emitting laser devices." Vertical-Cavity Surface-Emitting Lasers XV, J. K. Guenter, Chun Lei (editors), Proc. SPIE 7952, 79520B, 2011.

Copyright 2011 Society of Photo-Optical Instrumentation Engineers. One print or electronic copy may be made for personal use only. Systematic reproduction and distribution, duplication of any material in this paper for a fee or for commercial purposes, or modification of the content of the paper are prohibited.

DOI: 10.1117/12.873758

\begin{abstract}
Spin-controlled vertical-cavity surface-emitting lasers (VCSELs) have been intensively studied in recent years because of the low threshold feasibility and the nonlinearity above threshold, which make spin-VCSELs very promising for spintronic devices. Here we investigate the circular polarization dynamics of VCSELs on a picosecond time scale after pulsed optical spin injection at room temperature. A hybrid excitation technique combining continuous-wave (cw) unpolarized electrical excitation slightly above threshold and pulsed polarized optical excitation is applied. The experimental results demonstrate ultrafast circular polarization oscillations with a frequency of about $11 \mathrm{GHz}$. The oscillations last inside the first undulation of the intensity relaxation oscillations. Via theoretical calculations based on a rate equation model we analyze these oscillations as well as the underlying physical mechanisms.
\end{abstract}

Keywords: spin-VCSEL, spinoptoelectronic devices, polarization dynamics, spin-flip model

\section{INTRODUCTION}

The study of spin-controlled optoelectronic devices has revealed a considerable application potential based on the ability of transforming and modulating spin signals efficiently. These properties might enable signal storing, signal processing and communication applications. As a practical concept for spin-optoelectronic devices light emitting diodes (LEDs) with spin injection ${ }^{1-5}$ i.e. spin-LEDs, have attracted much attention. The major focus in the investigation of these devices is on the analysis of spin injection, spin relaxation and spin dephasing. Until now polarization degrees up to $32 \%$ have been achieved for spin-LEDs at room temperature (RT) ${ }^{6}$, which is however too low to satisfy the requirements for practical applications. Moreover, most spin-LEDs require strong external magnetic fields ensuring a high spin injection efficiency. Another drawback of spin-LEDs is the slow LED dynamics, which could limit their application in high speed signal processing and telecommunication.

In contrast, spin-controlled vertical-cavity surface-emitting lasers (spin-VCSELs) offer capabilities of high speed modulation due to laser dynamics and amplification of spin information based on their nonlinearity at threshold ${ }^{7,8}$. Furthermore spin-VCSELs may succeed their conventional counterparts with some properties such as low threshold and ultrafast polarization undulation capability due to their spin specificity. The implementation of first electrically pumped spin-VCSELs ${ }^{9,10}$ has recently established the perspective of spin-VCSELs for becoming practical spin-optoelectronic devices.

However, to fully exploit the above mentioned advantages, some drawbacks of spin-VCSELs such as linear polarization predetermination and irregular polarization undulations due to cavity anisotropies from laser material properties and fabrication must be carefully considered. A comprehensive understanding of the interplay between the spin excited 
emission and cavity anisotropies as birefringence and dichroism of the devices is necessary in order to develop efficient spin-VCSELs ${ }^{11,12}$. Upon cw excitation we have demonstrated that the linear polarization predetermination can be suppressed with spin polarized injection ${ }^{13,14}$. For the practical application, the temporal spin dynamics is of primary interest, so a comprehensive understanding of the complex interplay of spin dynamics, carrier density, photon density and devices parameters is highly desirable. Recently we have begun to study the spin dynamics of spin-VCSELs on a picosecond time scale upon pulsed spin injection at $\mathrm{RT}^{15}$. Here we analyze further the polarization dynamics of a commercial electrically pumped VCSEL with additional pulsed optical spin excitation at RT. Besides the experimental results we will also present the theoretical calculations revealing the connection between spin dynamics and linear polarized modes due to cavity anisotropies.

\section{POLARIZATION DYNAMICS OF VCSELS NEAR THE THRESHOLD}

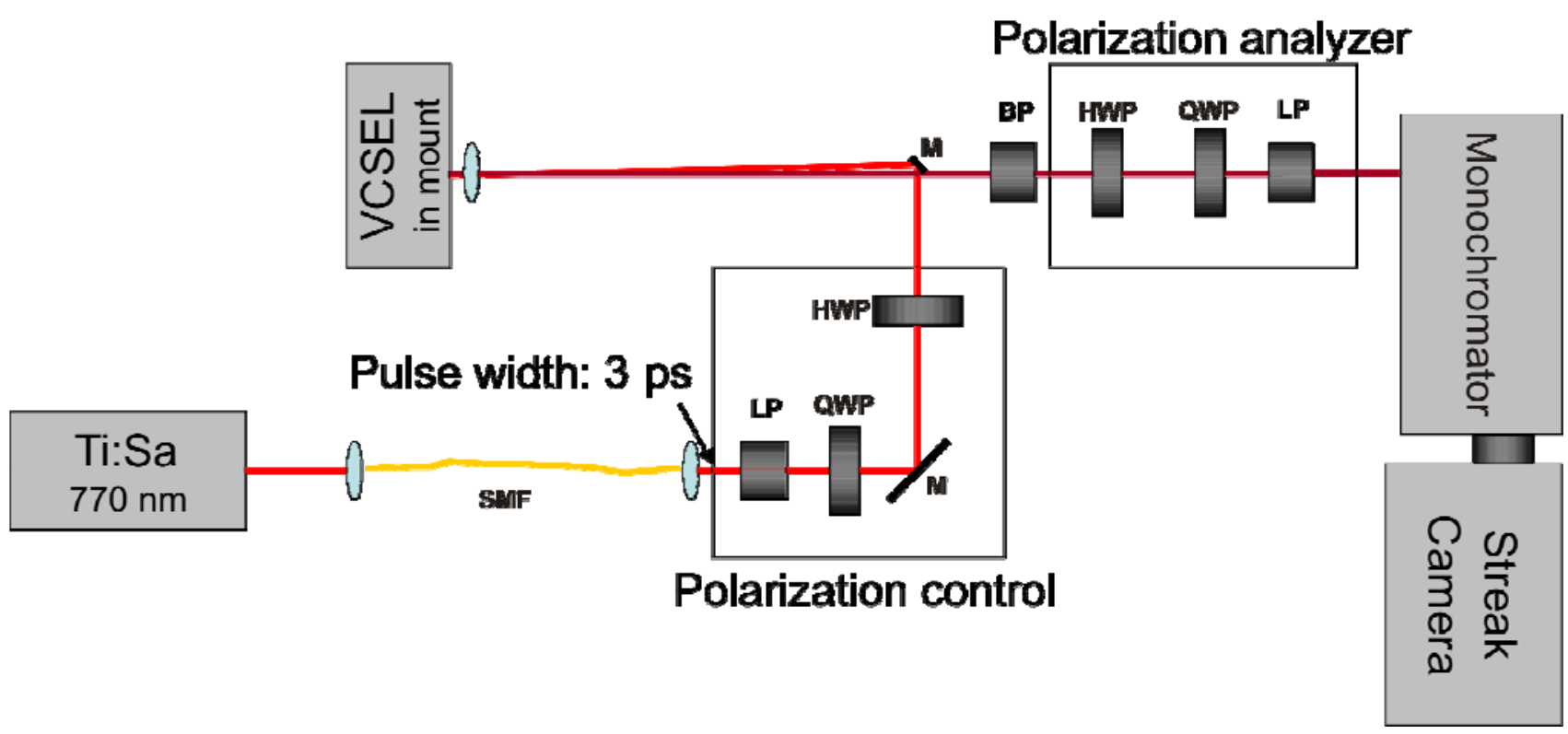

Fig 1. The experimental setup for the time-resolved polarization measurements: (M) mirror, (BP) bandpass, (HWP) half wave plate, (QWP) quarter wave plate, (LP) linear polarizer and (SMF) single mode fiber.

Nowadays the electrically pumped spin-VCSELs are only available at low temperature. That makes the experimental study on spin induced effects of such devices complicated and costly. Here we use a commercial electrically pumped VCSEL excited at RT, and analyze the polarization dynamics of its emission. As in our previous contribution a hybrid excitation scheme is employed, which combines cw unpolarized electrical excitation near the threshold and pulsed circularly polarized (circ. pol.) optical excitation. In this case carriers of both spin subbands are anisotropically excited due to the optical selection rules ${ }^{16}$. Corresponding to the handedness of the polarization the carrier density of one spin subband reaches the threshold earlier than the other, so only one circ. pol. laser mode is emitted while the other mode is suppressed. With an integrated measurement we have shown that the spin induced carriers in the active region determine the polarization of the output emission. In this letter we focus on the temporal dynamics of the polarization of the output emission.

The device under study is a commercial electrically pumped GaAs-QW VCSEL (Emcore, USA, type 8085-2010) with a emission wavelength of $830 \mathrm{~nm}$ at RT. The measurement setup is depicted in Fig. 1. The above mentioned pulsed circ. pol. optical pumping is offered by a mode-locked Ti:Sapphire laser, which emits laser pulses at $770 \mathrm{~nm}$ with a pulse width of $80 \mathrm{fs}$ and repetition rate of $75.5 \mathrm{MHz}$. The laser beam is at first coupled into a single mode fiber in order to 
remove an elliptical beam shape and then passes a polarization control composed of a linear polarizer and a quarter wave plate. After this the pulse width is extended to $3 \mathrm{ps}$ because of the dispersion from the single mode fiber. The polarization of the pulses can be precisely adjusted to any desired circular or linear polarization by the polarization control. The laser beam with the desired polarization is focused on the VCSEL surface nearly vertically. An extra half wave plate is used to eliminate the polarization distortion caused by two reflecting mirrors. The output emission is polarization and time resolved by a polarization analyzer and streak camera system. The electrically pumped VCSEL is operated near threshold and the average power of optical excitation was $150 \mathrm{~mW}$.

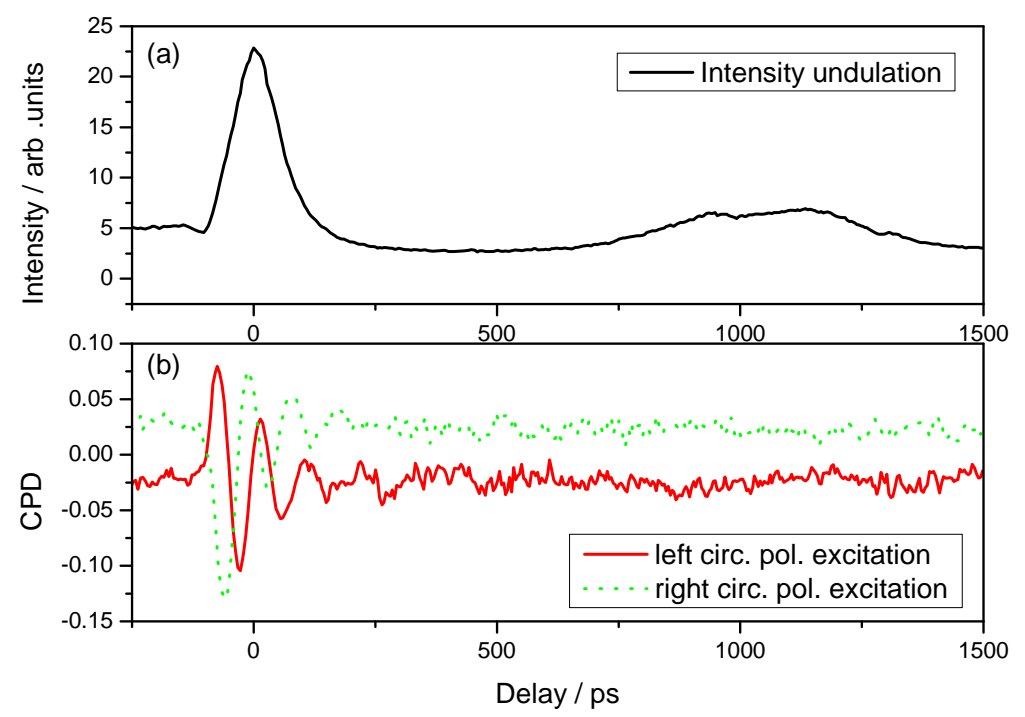

Fig 2. Temporal intensity undulation (a) and polarization dynamics under left (b, solid) and right (b, dashed) circ. pol. excitation.

Fig. 2 shows the undulation of the total intensity (a) and the calculated circular polarization degree (CPD) of the output emission under left and right circ. pol. excitation. The intensity undulation i.e. the intensity relaxation oscillation exhibits a first pulse of $150 \mathrm{ps}$ and a second pulse about $900 \mathrm{ps}$ later. In contrast to the intensity relaxation oscillation, the CPD dynamics shows a fast oscillation on a time scale shorter than $100 \mathrm{ps}$, which means an oscillation frequency higher than $10 \mathrm{GHz}$. The CPD peaks reach above 10\%. Corresponding to the handedness of the excitation polarization, the CPD dynamics begins with initial peaks of either positive or negative values. In both cases the CPD dynamics are strongly damped.

\section{THEORETICAL CALCULATIONS}

To understand the interplay between the spin polarized pumping and cavity anisotropy we perform here numerical calculations based on a spin flip model developed by San Miguel et al ${ }^{17,18}$. The laser modes are separately assumed as left and right circ. pol. with their complex amplitudes $E_{ \pm}$and corresponding intensities $I_{ \pm}=\left|E_{ \pm}\right|^{2}$. 


$$
\begin{aligned}
& \dot{E}_{ \pm}=\kappa(1+i \cdot \alpha)\left(N \pm m_{z}-1\right) E_{ \pm}-\left(\gamma_{a}+i \cdot \gamma_{p}\right) E_{\mp}+\xi_{ \pm} \sqrt{\beta \cdot \gamma \cdot\left(N \pm m_{z}\right)}, \\
& \dot{N}=\gamma\left[\eta_{+}+\eta_{-}-\left(1+I_{+}+I_{-}\right) N-\left(I_{+}-I_{-}\right) m_{z}\right], \\
& \dot{m}_{z}=\gamma\left(\eta_{+}-\eta_{-}\right)-\left[\gamma_{s}+\gamma\left(I_{+}+I_{-}\right)\right] m_{z}-\gamma\left(I_{+}-I_{-}\right) N .
\end{aligned}
$$

$\eta_{ \pm}$are pump terms for the spin up (right circ. pol.) and down (left circ. pol.) carrier reservoirs consisting of a background $\mathrm{CW}$ term describing the electrical excitation and a time dependent part describing the optical excitation, $\alpha$ is the linewidth enhancement factor, $\beta$ the spontaneous emission factor, $\xi_{ \pm}$the noise source, ${ }^{\kappa}$ the cavity decay rate, $\gamma$ the carrier density decay rate, $m_{z}$ the carrier spin magnetization with the decay rate $\gamma_{s}$ due to the spin flip processes, $\gamma_{p}$ the linear birefringence and the $\gamma_{a}$ the linear dichroism. This model can simplify the analysis of the underlying physical mechanisms in our case. Considering our experimental conditions and some calculations from earlier work on similar VCSEL devices we adapt a group of parameters to the model:

$$
\gamma=1.5 n s^{-1}, \gamma_{s}=40 n s^{-1}, \gamma_{p}=37 \mathrm{GHz}, \gamma_{a}=1.6 \pi \mathrm{GHz}, \kappa=300 n s^{-1}, \alpha=2, \beta=10^{-6} .
$$

Fig 3. shows the first result of our calculations. Here the spin unpolarized excitation is $105 \%$ of the threshold and the spin - up polarized excitation (a Gaussian pulse with pulse width of $3 \mathrm{ps}$, a peak energy of $4 \%$ of the output at threshold and a CPD of 0.5 ) is injected at $0 \mathrm{ps.} \mathrm{As} \mathrm{observed} \mathrm{in} \mathrm{our} \mathrm{experiment} \mathrm{during} \mathrm{the} \mathrm{intensity} \mathrm{relaxation} \mathrm{oscillation} \mathrm{a} \mathrm{CPD}$ oscillation can be found. The left (a, solid) and right (a, dotted) circ. pol. emission are plotted below the intensity relaxation (a, dashed). The peak value of the CPD oscillation reaches above $40 \%$.

With this spin flip model the experimental results are here very well reproduced, so is now possible to investigate the origin of the CPD oscillations. As a first step we calculate the dynamics of the carrier density in both spin subbands.
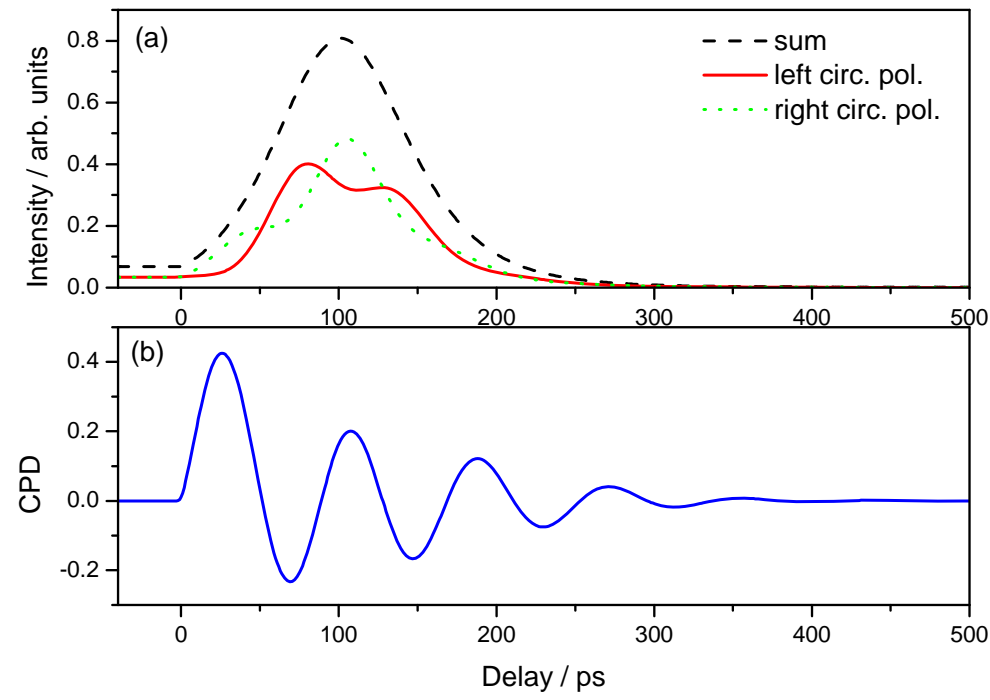

Fig. 3 Total intensity relaxation (a, dashed), the left (a, solid) and right (a, dotted) circ. pol. emission and CPD oscillation (b)

Fig 4. shows the dynamics of the carrier density in the active region and the polarization of the carriers in both spin subbands. After injection of the partly spin up polarized excitation we can observe that the carrier density of the spin up subband increases faster than of the spin down subband and reaches the threshold at first. After reaching the maximum 
of the carrier density the carrier density in the spin up subband begins to relax instantly and depletes within less than 200 ps after the incoming excitation pulse. It should be noted, that the carrier density of the spin down subband is smaller than that of the spin up subband due to the spin polarized excitation. It increases further slowly within about 50 ps while the spin up subband already begins to relax. According to the spin flip model this phenomenon can be explained with two mechanisms: first, by the combination between spin relaxation and spin dephasing, which reduces the difference between both spin subbands. This enters into the model as spin flip rate. Second, by the cavity anisotropy, which couples the left and right circ. pol. photons, and introduces spin down carriers in the active region. The coupling between the two relaxations of both spin subbands results in the CPD oscillations. In Fig 4. (bottom) the polarization of the carriers in the active region is also depicted. It reaches a maximum of about $2.5 \%$ then relaxes rapidly to 0 . Remarkably, slightly above the threshold the CPD of the emission can be $40 \%$ even with only $2.5 \%$ polarization of the carriers in the active region. Next, we investigate the two mechanisms separately.

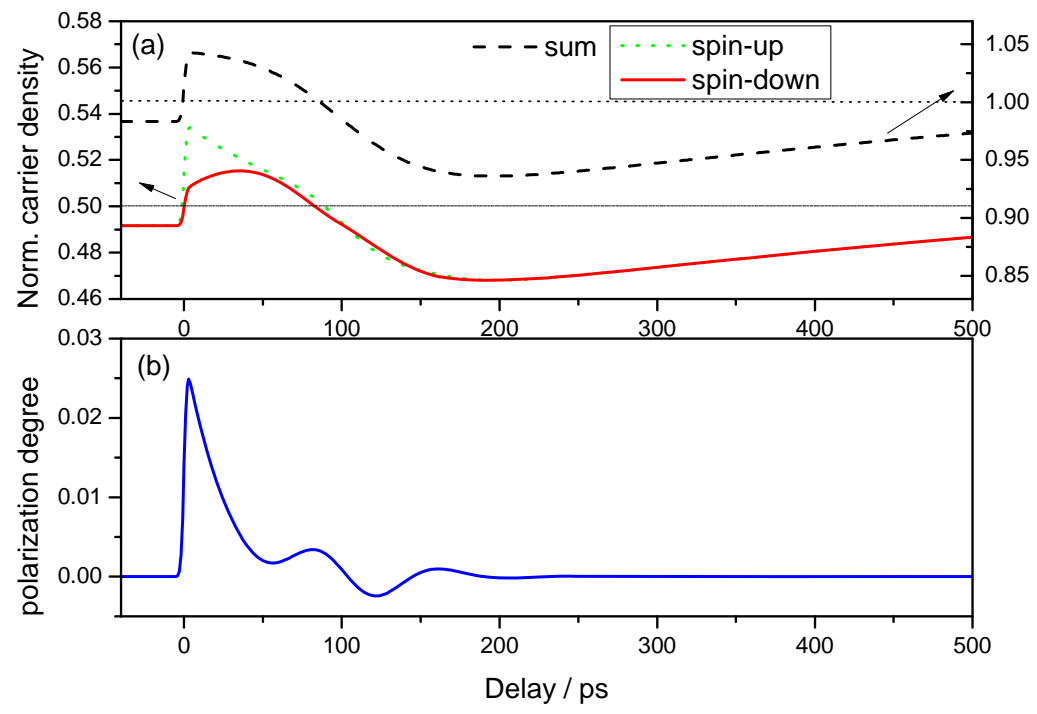

Fig 4. The normalized carrier density in active region (a, dashed), the carrier density of spin up (a, dotted) and spin down (a, solid) subband. The polarization of carriers (b) is calculated from the carrier density in both spin subbands.

To verify the dependence of the CPD dynamics on the spin flip rate and the cavity anisotropy, calculations with varying spin flip rate and birefringence were performed. Fig 5. shows the CPD dynamics with different spin flip rates (from 10 to $90 \mathrm{GHz}$ ). With high spin flip rates the CPD oscillations show smaller peak values. According to the Fourier analysis the frequency of the CPD oscillations is constant. That reveals the weak dependence of the CPD dynamics on the spin flip rate in this regime. 

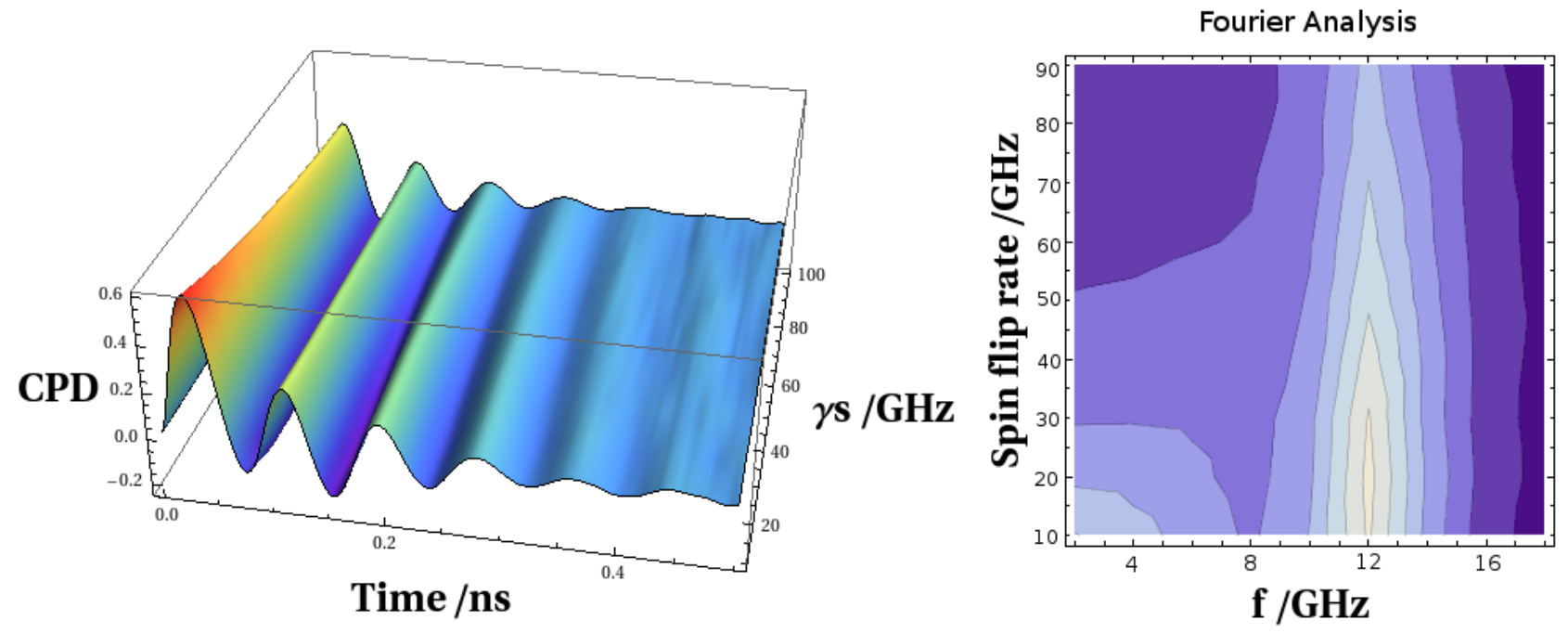

Fig 5. The CPD oscillations with increasing spin flip rate and the corresponding Fourier analysis

Fig 6. shows the results for varying birefringence $(5 \sim 85 \mathrm{GHz})$. Comparing to the calculations with varying spin flip rate there are clear difference to observe. With increasing birefringence the CPD dynamics shows oscillation with higher frequency. At $5 \mathrm{GHz}$ the $\mathrm{CPD}$ oscillation is almost suppressed. Only one peak is visible.

Both theoretical calculations show that the experimental CPD oscillation frequency has a negligible dependence on the spin flip rate in the given regime. The coupling between cavity anisotropy (birefringence) and carriers is the main cause of the CPD oscillations. That provides a possibility to control the oscillation frequency of CPD oscillations
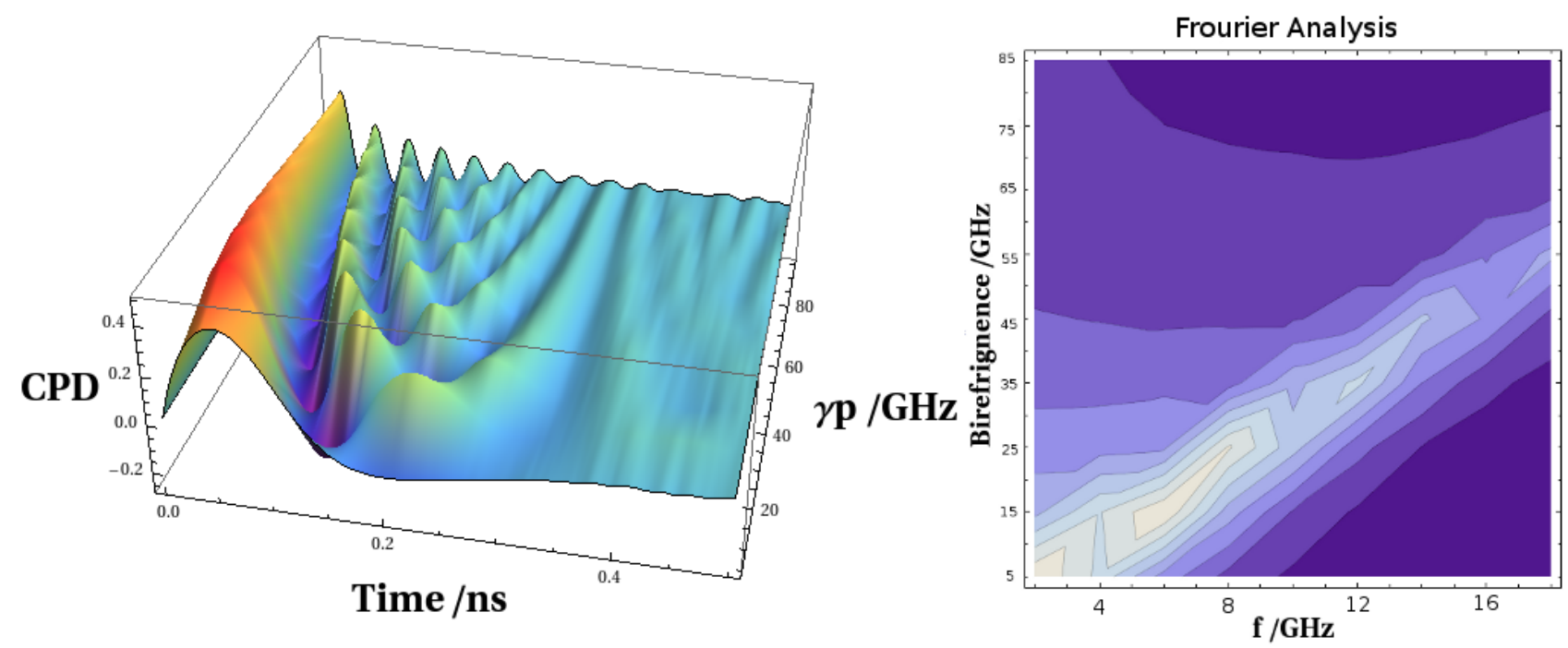

Fig. 6 The CPD oscillations with increasing birefringence and the corresponding Fourier analysis 


\section{CONCLUSION}

In summary, we have investigated the polarization dynamics of an electrically pumped vertical-cavity surface-emitting laser with optically spin induced carriers at room temperature. In our experiment spin induced oscillations of the circular polarization degree have been observed during the emitted VCSEL pulse after partly spin polarized excitation slightly above the threshold. With a spin flip model we reproduced the experimental results and analyzed the dynamics of the carrier densities in both spin subbands. The observed polarization oscillation is a consequence of the coupling of carriers in both spin subbands in active region and the cavity anisotropy. The frequency of such dynamics depends strongly on the birefringence in the cavity anisotropy, which should be carefully controlled, to exploit the potential of spin VCSEL devices

\section{ACKNOWLEDGMENTS}

The authors thank the Deutsche Forschungsgesellschaft (DFG) for support within the Sonderforchungsbereich 491.

\section{REFERENCES}

[1] Fiederling, R., Keim, M., Reuscher, G., Ossau, W., Schmidt, G., Wagg, A. and Molenkamp, L.W., "Injection and detection of a spin-polarized current in a light-emitting diode." Nature 402, 787-790 (1999)

[2] Ohno, Y., Young, D.K., Beschoten, B., Matsukura, F., Ohno, H. and Awschalom, D.D., "Electrical spin injection in a ferromagnetic semiconductor heterostructure." Nature 402, 790-792 (1999)

[3] Zhu, H., Ramsteiner, M., Kostial, H., Wassermeier, M., Schönherr, H.P. and Ploog, K. “Room-Temperature Spin Injection from Fe into GaAs." Phys. Rev. Lett. 87, 1-4 (2001)

[4] Hanbicki, A.T., J onker, B.T., Itskos, G., Kioseoglou, G., and Petrou, A. "Efficient electrical spin injection from a magnetic metal/tunnel barrier contact into a semiconductor." Appl. Phys. Lett. 80, 1240 (2002)

[5] Gerhardt, N.C., Hövel, S., Brenner, C., Hofmann, M.R., Lo, F.-Y., Reuter, D., Wieck, A.D., Schuster, E., Keune, W. and Westerholt, K., "Electron spin injection into GaAs from ferromagnetic contacts in remanence." Appl. Phys. Lett. 87, 032502 (2005)

[6] Jiang, X., Wang, R., Shelby, R.M., Macfarlane, R.M., Bank, S.R., Harris, J.S., and Parkin, S.S., "Highly Spin-Polarized Room-Temperature Tunnel Injector for Semiconductor Spintronics using MgO(100)." Phys. Rev. Lett. 94, 056601 (2005)

[7] Hövel, S., Hofmann, M.R., Yang, J., Reuter, D. and Wieck, A.D., "Spin controlled opticlly pumped vertical cavity surface emitting laser." Electronics Letters 41, 251 (2005)

[8] Gerhardt, N.C., Hövel, S., Hofmann, M.R., Yang, J., Reuter, D. and Wieck, A.D., "Enhancement of spin information with vertical cavity surface emitting lasers." Electronics Letters 42, 88 (2006)

[9] Holub, M., Shin, J., Saha, D. and Bhattacharya, P., “Electrical Spin Injection and Threshold Reduction in a Semiconductor Laser." Phys. Rev. Lett. 98, 1-4 (2007)

[10] Basu, D., Saha, D., Wu, C.C., Holub, M., Mi, Z. and Bhattacharya, P., "Electrically injected InAs/GaAs quantum dot spin laser operating at $200 \mathrm{~K} . "$ Appl. Phys. Lett. 92, 091119 (2008)

[11] Sondermann, M., Weinkath, M. and Ackemann, T., "Polarization switching to the gain disfavored mode in vertical-cavity surface-emitting lasers." IEEE J. Quantum Electron. 40, 
97-104 (2004)

[12] Ackemann, T., and Sondermann, M., "Characteristics of polarization switching from the low to the high frequency mode in vertical-cavity surface-emitting lasers." Appl. Phys. Lett. 78, 3574 (2001)

[13] Ho vel, S., Bischoff, A., Gerhardt, N.C., Hofmann, M.R., Ackemann, T., Kroner, A. and Michalzik, R., "Optical spin manipulation of electrically pumped vertical-cavity surfaceemitting lasers." Appl. Phys. Lett. 92, 041118 (2008)

[14] Ho vel, S., Gerhardt, N.C., Hofmann, M.R., Lo, F.-Y., Reuter, D., Wieck, A.D., Schuster, E., Wende, H., Keune, W., Petracic, O. and Westerholt, K., "Electrical detection of photoinduced spins both at room temperature and in remanence." Appl. Phys. Lett. 92, 242102 (2008)

[15] Li, M.Y., Jaehme, H., Soldat, H., Gerhardt, N.C., Hofmann, M.R. and Ackemann, T., "Birefringence controlled room-temperature picosecond spin dynamics close to the threshold of vertical-cavity surface-emitting laser devices." Appl. Phys. Lett. 97, 191114 (2010)

[16] Dyakonov, M. and Perel, V. Optical Orientation, Elsevier Science Publishers B.V., (1984)

[17] San Miguel, M., Feng, Q. and Monoley, J.V. "Light-polarization dynamics in surfaceemitting semiconductor lasers." Phys. Rev. A 52, 1728-1739. (1995)

[18] Gahl, A., San Miguel, M. and Balle, S. "Polarization dynamics of optically pumped VCSELS." IEEE J. Quantum Electron. 35, 342-351 (1999) 